\title{
Numerical Investigation on Heat Transfer Characteristics of Single Jet Impingement Cooling
}

\author{
Hong Zifeng ${ }^{1}$, Zhao Jing ${ }^{1, a^{*}}$, Li Yanjun ${ }^{2}$, Yang Longbin ${ }^{2}$, Zhu Weibing $^{3}$ \\ ${ }^{1} \mathrm{NO} .703$ Research Institute of CSIC, Harbin, 150078, China \\ ${ }^{2}$ College of Power and Energy Engineering, Harbin Engineering University, Harbin, 150001, China \\ 3 \\ College of Aerospace and Civil Engineering, Harbin Engineering University, Harbin, 150001, China \\ azhaojingcopaee@163.com
}

Keywords: Single Jet; Impingement Cooling; Heat Transfer Characteristics

Abstract. Numerical computation with Transition $k-k l-\omega$ model is conducted to investigate the heat transfer characteristics for the single jet impingement cooling. The effects of the jet Reynolds number and the impingement spacing (jet-to-surface spacing, $Z n / D$ ) on heat transfer characteristic are researched. Results show that the numerical predictions are in good agreement with the analytical solution and experiment data. The maximum value of the Nusselt number is located in $X / D=0.75$. The bigger Reynolds number is, the better cooling effect is. There exists an optimal impingement spacing for heat transfer effect, the Nusselt number could reach the highest value in $Z n / D=4$.

\section{Introduction}

As an efficient heat exchange technology, the impingement cooling has been widely applied in the hot end components (eg. turbine blade) of gas turbine engine and enhancing cooling of the other thermal power installations. Metzger et al. ${ }^{[1-3]}$ firstly investigated the principle of the impingement cooling, and they summarized the influence factors of heat transfer of porous vertical impingement cooling plate. Osama ${ }^{[4]}$ analyzed the flow field distribution of jet impingement for the single jet impinging solid surface vertically. Florschuetz et al. ${ }^{[5]}$ studied the effect of cross flow on Nusselt number. With the rapid development of computer technology, numerical simulation has becomes a powerful tool to study the jet impingement cooling. The heat transfer characteristics of impingement cooling have been investigated by a large number of researchers. However, the studies about the influence factors of heat transfer effect of the impingement cooling are not sufficient. Therefore, in this paper, we numerically simulate the heat transfer characteristics of the single jet impingement cooling, and analyze the influence of the jet Reynolds number and the impingement spacing (jet-to-surface spacing) on the heat transfer effect.

\section{Model equations}

The continuity, momentum and energy equations are shown as follows

$$
\begin{aligned}
& \frac{\partial \rho}{\partial t}+\nabla \cdot(\rho \mathbf{u})=0 \\
& \frac{\partial}{\partial t}(\rho \boldsymbol{u})+\nabla \cdot(\rho \mathbf{u} \boldsymbol{u})=\nabla \cdot\left(-p \delta+\mu\left(\nabla \mathbf{u}+(\nabla \mathbf{u})^{T}\right)\right)+S_{M} \\
& \frac{\partial}{\partial t}\left(\rho h^{*}\right)-\frac{\partial p}{\partial t}+\nabla \cdot\left(\rho \boldsymbol{u} h^{*}\right)=\nabla \cdot(\lambda \nabla T)+\nabla \cdot\left(\mu\left(\nabla \mathbf{u}+(\nabla \boldsymbol{u})^{T}\right)-\frac{2}{3} \nabla \cdot \mathbf{u} \delta \boldsymbol{u}\right)+S_{E}
\end{aligned}
$$

where $S_{M}$ are the momentum source term, $S_{E}$ are the energy source term, and $h^{*}$ are the total enthalpy. 


\section{Calculation conditions}

The calculation model of single jet is presented in Fig. 1(a). We select jet diameter $D=1.2 \mathrm{~mm}$. The symmetry boundary is used for reducing calculated amount. The mass flux is given at the jet inlet (the mass flux $m=\operatorname{Re} \pi D \mu / 4$ ), and the total temperature is set to $300 \mathrm{~K}$. We set four outlet orientation and adopt the pressure outlet. A constant heat flux of $20 \mathrm{KW} / \mathrm{m}^{2}$ is used for target wall, and the other walls are set to adiabatic and no-slip boundary conditions. Fig. 1(b) shows the mesh generation, and the boundary layer mesh is generated on the fluid-solid interface. We employ the pressure-based steady state solver and the transition $k-k l-\omega$ model. The enhanced wall treatment is used for wall treatment, and SIMPLE algorithm is adopted for pressure-velocity coupling. The discretization schemes of all physcial quantities are second order upwind scheme. The residual error is set to $10^{-4}$. Due to velocity of gas is smaller than $0.3 \mathrm{Ma}$, the assumption of incompressible fluid is reasonable. The material of solid zone is selected to carbon steel. We selecte four grid numbers: 0.27 million, 0.45 million, 0.67 million and one million. We find that the grid number of 0.67 million is enough to satisfy the computing requirement.

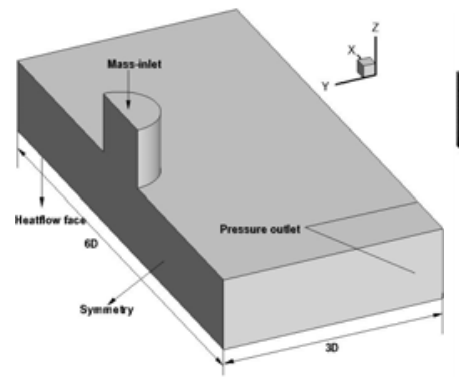

(a) calculation model

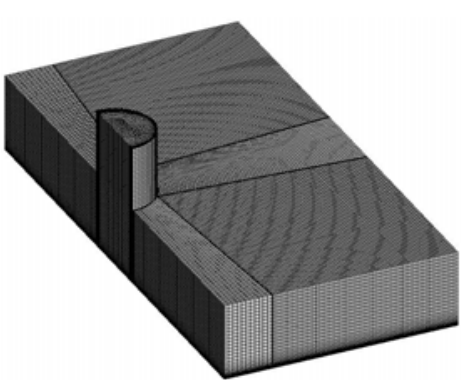

(b)mesh

Fig. 1 Schematic diagram of calculation model

\section{Results and Discussions}

Fig. 2 shows the Nusselt number $(N u)$ on the target wall and velocity distribution on the section along $\mathrm{X}$ direction of center jet in $R e=5000$. It can be seen that the distribution of $N u$ is radiation, and the variation of $N u$ is close to function of radius. $N u$ in the center of stationary point is smaller than that the downstream region. The impingement flow is a sort of jet flow, and it forms the bounded jet because the jet flow is obstructed by the target wall in the direction of forward motion, which is different from free jet. It can be seen that, from corresponding velocity distribution, the axial velocity decreases all the way after jet flow erupts from jet hole. The flow is in jet flow core area and impinges wall (the jet flow is not fully developed), and then the jet flow rotates in free shear layer and wall boundary layer and enters into break over region (it also known as stationary point region). In the stationary point region, the axial velocity ( $z$ direction in Fig.2) and the component of velocity of perpendicular to axis do not equal zero. The closer the distance of jet flow and target wall is, the smaller axial velocity is, but the larger the component of velocity of $X Y$ plane is. In this region, the jet flow deflects $90^{\circ}$ and enters into the wall jet area (fluid flow is parallel to target wall in this region).

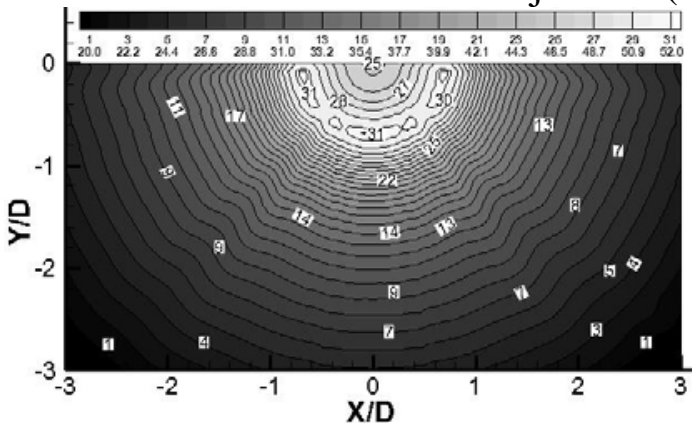

(a) Nusselt number

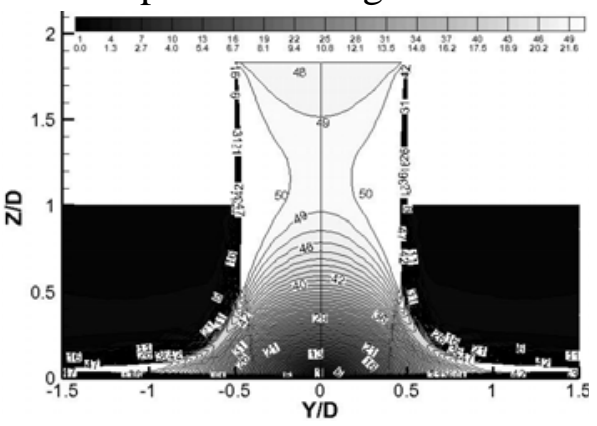

(b) velocity

Fig. 2 Nusselt number and velocity distribution in $R e=5000$ 
Fig. 3 presents the local Nusselt number on the target wall at the different jet Reynolds number. With the increasing of Reynolds number, the Nusselt number increases and the heat transfer effect is enhanced. In each Reynolds number, the local Nusselt number is not monotonously change along the target wall, but it has a peak distribution in the range of Reynolds number in this paper. The maximum value is located in $X / D=0.75$, and the larger $R e$ is, the more obvious peak distribution is. The heat transfer characteristic is closely related to the flow characteristic. The boundary layer of stationary point region is usually laminar boundary layer and extremely thin, so the coefficient of heat transfer is large. Once the stationary point region end, the boundary layer turns into the turbulent boundary layer in the wall jet region and the Nusselt number suddenly rises. One can conclude that the transition position is about located in $X / D=0.75$ where is the position of the end of the stationary point region and the start of the wall jet region.

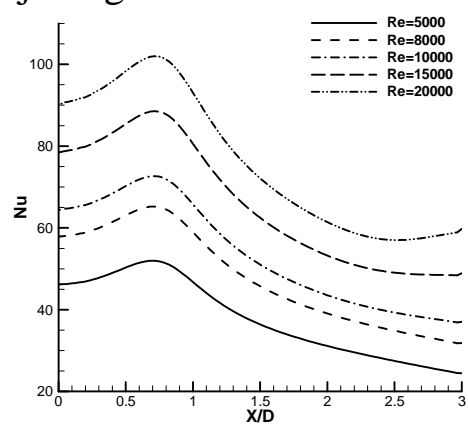

Fig. 3 Local Nusselt number on the target wall at the different Reynolds number

The local Nusselt number reflects the variation trend of local cooling effect in impingement cavity, which is contributed to understand the distribution of heat transfer and flow field. In the practical engineering application, however, the global cooling effect is also concerned, namely, the average Nusselt number. The simulated average Nusselt number on the target wall is compared with analytical solution and experiment data at the different Reynolds number in Fig. 4. The numerical predictions are in good agreement with the analytical solution and experiment data. When Reynolds number is larger than 8000 , especially, our results have maximum relative errors of $1 \%$ with analytical solution and $5 \%$ with experiment data. This illustrates the average heat transfer characteristic of target wall structure can be excellently predicted by using the transition $k-k l-\omega$.

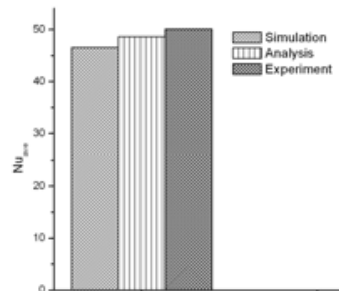

(a) $R e=5000$

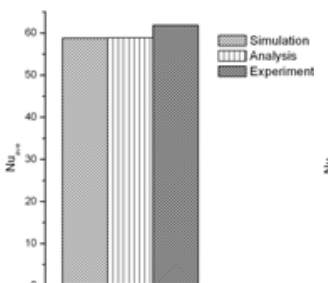

(b) $R e=8000$

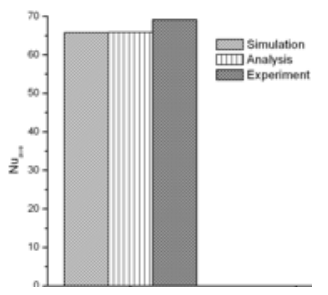

(c) $R e=10000$

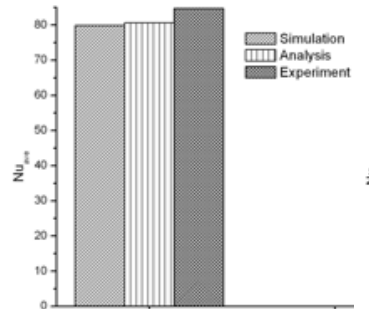

(d) $R e=15000$

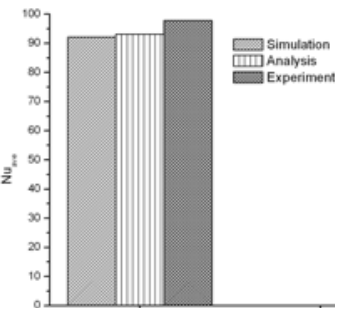

(e) $R e=20000$

Fig.4 The average Nusselt Number at the different Reynolds number

Fig. 5 provides the variation of $N u$ with impingement spacing $\left(Z_{n} / D\right)$ at the different Reynolds number. The maximum Nusselt Number presents in $Z_{n} / D=4$, that is to say the cooling effect is the best in here. The distribution of $N u$ is basically consistent in $Z_{n} / D=1$ and $Z_{n} / D=2$. In $R e=5000-10000$, the distribution of $N u$ in $Z_{n} / D=3$ is basically consistent with in $Z_{n} / D=1$ (and $Z_{n} / D=2$ ), but the former is apparently higher than the latter in $R e=15000$ and 20000 . When $R e=5000$ and 8000, the cooling effect $(N u)$ in $Z_{n} / D=7.5$ has less difference with in $Z_{n} / D=4$, while the $N u$ of the former is obviously lower 
than the latter in $R e>10000$. From Fig. 5 it can be also seen that the transition position in the stationary point region migrates by changing the impingement spacing. The larger impingement spacing is, the closer transition position with the central position stationary point of is.

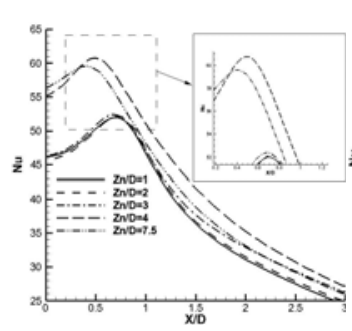

(a) $\operatorname{Re}=5000$

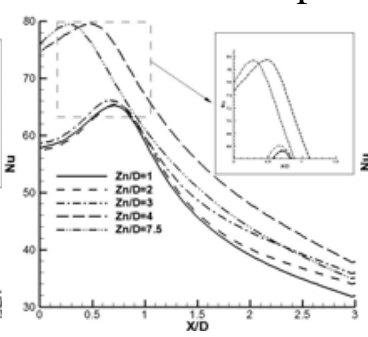

(b) $R e=8000$

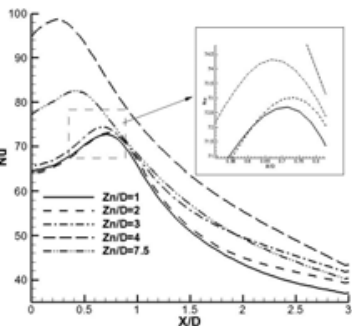

(c) $R e=10000$

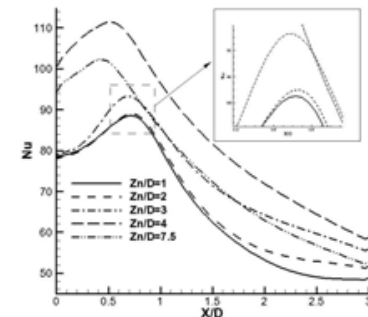

(d) $R e=15000$

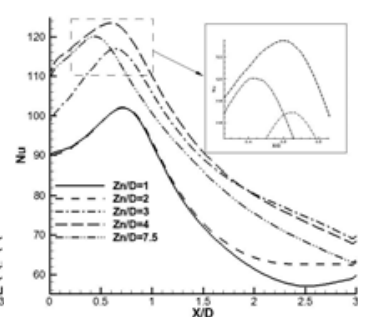

(e) $R e=20000$

Fig. 5 The variation of $\mathrm{Nu}$ with impingement spacing at the different Reynolds number

\section{Conclusion}

In this work, we investigate the heat transfer characteristics for single jet impingement cooling by using transition $k-k l-\omega$ model. The results indicate that the numerical predictions are in good agreement with the analytical solution and experiment data. Especially, our results have maximum relative errors of $1 \%$ with analytical solution and 5\% with experiment data in $R e>8000$. $N u$ has a peak distribution in the range of $R e$ in this paper, and the maximum value is located in $X / D=0.75$. The larger $R e$ is, the more obvious peak distribution is. The bigger $R e$ is, the better cooling effect is. With the increasing of impingement spacing, $N u$ increases and then reduces. The optimum impingement spacing is $Z_{n} / D=4$.

\section{References}

[1] Metzger, D E, Florschuetz L W, Takeuchi D I, et al., Heat transfer characteristics for inline and staggered arrays of circular jets with crossflow of spent air, Journal of Heat Transfer. 101 (1979) 526-531.

[2] Metzger D E, Korstad R J, Effects of crossflow on impingement heat transfer, Journal of Engineering for Gas Turbines and Power. 94 (1972) 35-41.

[3] Metzger D E, Yamashita T, Jenkins C W, Impingement cooling of concave surfaces with lines of circular air jets, Journal of Engineering for Gas Turbines and Power. 91 (1969) 149-155.

[4] Al-aqal O M A, Heat transfer distributions on the walls of a narrow channel with jet impingement and cross flow, University of Pittsburgh, 2003.

[5] Florschuetz L W, Su C C, Effects of crossflow temperature on heat transfer within an array of impinging jets, Journal of heat transfer. 109 (1987) 74-82.

[6] Martin H. Heat and mass transfer between impinging gas jets and solid surfaces, In: Advances in heat transfer. New York, Academic Press, Inc., 1977, pp. 1-60.

[7] Hrycak P, Lee D T, Gauntner J W, et al, Experimental flow characteristics of a single turbulent jet impinging on a flat plate, National Aeronautics and Space Administration, 1970. 